\title{
Reflection and reviewers appreciation
}

\author{
Yoram Reich
}

Published online: 21 December 2011

(C) Springer-Verlag London Limited 2011

Two years after the restructuring of RED, it is appropriate to reflect on the process and its outcome. The details of the restructuring could be found in a previous editorial (V21, no. 2) and included mainly the addition of the area editors that are responsible for submission handling within particular areas of expertise. Two years later, the editorial board team certainly provides better service to authors. We are gradually approaching our targets for timely review of papers but we are not yet there; some papers still take too long to review, and some papers are returned to authors with two reviews only. Nevertheless, feedback from authors suggests that by and large, we do provide good reviews that are written by reviewers that are experts in the subject matter of the papers.

Due to these improvements, we have moved from a situation where we had just the needed number of papers to publish issues, to a situation where he have a queue of papers waiting to be published on the web (Online First ${ }^{\mathrm{TM}}$ ). We anticipate that this queue will increase slightly even though last year, we increased the number of pages per issue from about 65 to 80 and we intend to extend this page count further next year. In order to support this operation and continue to improve the quality of the journal as we did last year, next year will be devoted to renew and strengthen the editorial board and improve the process of selecting reviewers. We will also develop better guidelines for reviewers so that the reviews will help editors make their decisions and authors will get the guidance they need for improving their papers.

This new issue marks the ending of 2011 and it is time to thank all the reviewers who contributed their precious

Y. Reich $(\bowtie)$

Tel Aviv, Israel

e-mail: yoram@eng.tau.ac.il time to review papers and provide valuable comments to authors. This effort maintains and improves the high quality of the journal. The following are the reviewers, other than editorial board members, who contributed their reviews in 2011:

Sofiane Achiche

Robin Adams

Saeema Ahmed-Kristensen

Albert Albers

Janet Allen

Edward Anderson

Claudiano Araujo

Simon Austin

Gideon Avigad

Terry Bahill

Linden J. Ball

Glenn Ballard

Sudhirkumar Barai

Catherine Barnes

David Ben-Arieh

Yakov Ben-Haim

Herbert Birkhofer

Janneke Blijlevens

David Blockley

Casper Boks

Julian Booker

Jean Francois Boujut

Rob Bracewell

Jürgen Branke

Daniel Brissaud

Willem Bronsvoort

Tyson Browning

Stuart Burgess

Jerry Busby

Jaime Camelio 
Matthew Campbell

Marco Cantamessa

Carlos Cardoso

H.P. Casakin

Kevin Caskey

Sylvie Castagne

Yuh-Min Chen

Chun-Hsien Chen

Songlin Chen

Wei Chen

Jahau Chen

Rae Cho

Yih Tng Chong

P. John Clarkson

Pedro Company

Clark Cory

Nathan Crilly

Richard Crowder

Steve Culley

Arnaud De Grave

Roland De Guio

Oliver De Weck

Kalyanmoy Deb

Elies Dekoninck

Peter Demian

Micael Derelöv

Snelders Dirk

Andy Dong

Kees Dorst

Xiaoping Du

Gui-Jiang Duan

Chris Earl

Steven Eppinger

Ozgur Eris

Tijen Ertay

Gualtiero Fantoni

Gary Fedder

Joëlle Forest

Maarten Franssen

Renate Fruchter

Erica Fuchs

Ioannis Fudos

Kikuo Fujita

Fei Gao

John Gershenson

Matt Giess

Ashok Goel

Tushar Goel

Gabriela Goldschmidt

Paul Grogan

Marin Guenov

C. Dominik Guess

Jian Guo
Peter Heisig

Paul Hekkert

Lex Hendriks

Jeffrey Herrmann

Matthias Heymann

Christian Hicks

Ben Hicks

Raymond Holt

Katja Hölttä-Otto

Imre Horvath

Thomas Howard

Gregory Howell

Hsu-Fang Hung

Abdessamad Imine

Bipin Indurkhya

Aminul Islam

Santosh Jagtap

Jianxin Roger Jiao

Yan Jin

Nitin Joglekar

Michael Johnson

Jeff W.T. Kan

Maya Kaner

Arie Karniel

Konstantinos Katsikopoulos

Akin Kazakçi

David Kazmer

Turkka Keinonen

Yves Keraron

Warren Kerley

Li-Pheng Khoo

Harrison Kim

Sang-Gook Kim

Kyoung-Yun Kim

Yoshinobu Kitamura

Maaike Kleinsmann

Hideki Kobayashi

Fb Kong

Lauri Koskela

Gül Kremer

Ehud Kroll

Tolga Kurtoglu

C. Kwong

Kristina Lauche

Pascal Le Masson

Yu-Cheng Lee

Jeremy Legardeur

Kemper Lewis

Li Lin

Ying Liu

Ying-Chieh Liu

Belinda Lopez-Mesa

Peter E. D. Love 
Anthony Luscher

Weiyin Ma

Ade Mabogunje

Elina Madetoja

Christopher Magee

Richard Malak Jr.

Jane Malin

Johan Malmqvist

Creusen Marielle

Peter Matthews

Kurt Matzler

Maik Maurer

Daniel Mcadams

Janet Mcdonnell

Alison Mckay

Christoph Meinel

Andres Melo

Jurgen Mihm

Greg Mocko

Jose Molina

Ira Monarch

Francesca Montagna

A. Morris

Amiram Moshaiov

Damien Motte

James Moultrie

Elena Mulet

Sabina Muschik

Yukari Nagai

Masuru Nakano

Aydin Nassehi

Margareta Norell Bergendahl

William Notz

Sharad Oberoi

Josef Oehmen

Kevin Otto

Stig Ottosson

Mohamed Zied Ouertani

Belkacem Ould Bouamama

Rivka Oxman

Robert Paasch

Jitesh Panchal

Chris Paredis

Michael Pasqual

Qingjin Peng

Ruben Perez

Kin-Leong Pey

Vesna Popovic

Ravi Rangan

William Regli

Gerard Renardel de Lavalette

John Renaud

Donna Rhodes
Leenders Roger

Carolyn Rose

Utpal Roy

Stephan Rudolph

Donald Saari

Pierre Sachse

Rafael Sacks

Sa'ed Salhieh

Filippo Salustri

Prabir Sarkar

Michael Schabacker

Harald Schaub

Rudolf Scheidl

Rick Schifferstein

Anne-Françoise Schmid

Linda Schmidt

Jan Schoormans

Chris Schunn

Michael Scott

Carolyn Seepersad

Armin Shmilovici

Frido Smulders

Durward Sobek, II

Martin Stacey

George Stiny

Robert Stone

Richard Storch

Stefan Strohschneider

Joshua Summers

Shun Takai

Dunbing Tang

Derrick Tate

Toshiharu Taura

Graham Thompson

Sof Thrane

Deborah Thurston

Ashutosh Tiwari

Jan Treur

Tommy Tucker

Irem Tumer

Yasushi Umeda

Ingrid Utne

Ibo Van De Poel

Qi Van Eikema Hommes

Pieter Van Langen

Thomas Van Rompay

Jan Vandenbrande

Noe Vargas Hernandez

Srinivasan Venkataraman

Pieter Vermaas

Giovanna Vianello

S. Vinodh

Willemien Visser 
Gary Wang

Sandro Wartzack

Christian Weber

Benoit Weil

Daniel Whitney

Margaret Wiecek

Paul Wormald

Teresa Wu

David Wynn

Xun $\mathrm{Xu}$

Kai Yang
Maria Yang

Deng Yi-Min

Masaharu Yoshioka

Winfried Zanker

Roman Zavbi

Wim Zeiler

Yong Zeng

Xuan Zha

Linda (Lianfeng) Zhang

Marc Zolghadri 